\title{
Letter
}

\section{A rotating and magnetized three-dimensional hot plasma equilibrium in a gravitational field}

\author{
Peter J. Catto ${ }^{1} \dagger$ and Sergei I. Krasheninnikov ${ }^{2}$ \\ ${ }^{1}$ Plasma Science and Fusion Center, Massachusetts Institute of Technology, Cambridge, MA 02139, USA \\ ${ }^{2}$ Department of Mechanical \& Aerospace Engineering, University of California San Diego, La Jolla, \\ CA 92093, USA
}

(Received 6 January 2015; revised 27 February 2015; accepted 2 March 2015; first published online 1 April 2015)

A rotating and magnetized three-dimensional axisymmetric equilibrium for hot plasma confined by a gravitational field is found. The plasma density and current can exhibit strong equatorial plane localization, resulting in disk equilibria with open magnetic field lines. The associated equatorial plane pinching results in magnetic field flaring, implying a strong gravitational squeezing of the plasma carrying ambient magnetic field lines toward the gravitational source. At high plasma pressure, the magnetic field becomes strongly radial outside the disk. The model predicts the rotation frequency bound, the condition for a plasma disk, and the requirement for strong magnetic field flaring.

\section{Introduction}

Analytic axisymmetric three-dimensional confined magnetized plasma equilibria under the combined influence of gravitational and centrifugal forces are of interest for astrophysical and planetary applications, and in particular for magnetized accretion disks. We present a three-dimensional analytic equilibrium solution for hot, toroidally rotating, gravitationally and magnetically confined plasma. In addition to satisfying Ampere's law, quasineutrality, continuity, and momentum conservation, our solution is consistent with the kinetic constraints on a drifting Maxwellian that are imposed by the Fokker-Planck equation (Hinton and Wong 1985; Catto et al. 1987; Helander 2014).

Early magnetohydrodynamic (MHD) considerations retaining gravity (Chandrasekhar 1956) assumed strict incompressibility with constant plasma density. Later ideal MHD formulations treating gravity require poloidal flow (Prasanna et al. 1989; McClements and Thyagaraya 2001; Throumoulopoulos and Tasso 2001), which is not permitted kinetically. In addition, Throumoulopoulos and Tasso (2001) assume that the density is a flux function and considers only dipole solutions, while Prasanna et al. (1989) do not treat the frozen-in field flow constraint and try to satisfy Gauss's law rather than quasineutrality. In addition, these treatments normally retain a toroidal magnetic field that we assume to vanish since it is not required to maintain equilibrium. Works by Lovelace et al. (1986) and Ogilvie (1997) are noteworthy because they focus on the thin disk limit with no 
poloidal flow or toroidal magnetic field, assume a polytropic or adiabatic equation of state relating the pressure and density. Our magnetic equilibrium model is even simpler and employs an isothermal relation between the pressure and density with the temperature being a flux function as required by kinetic theory. We seek axisymmetric self-similar solutions that lead to an ordinary second-order nonlinear differential equation retaining gravity, toroidal rotation, and plasma pressure. We find flared, open magnetic field line solutions rather than dipole magnetic field lines closing at the localized gravitational source (Prasanna et al. 1989; McClements and Thyagaraya 2001; Throumoulopoulos and Tasso 2001; Ghanbari and Abbassi 2004). We expect the equilibrium that we find will be useful in setting up global simulations to investigate magneto-rotational stability in magnetized accretion disks (Velikhov 1959; Balbus and Hawley 1991). Perhaps, shedding light on the detailed mechanisms by which mass accretion occurs at a black hole while momentum is transported outward.

\section{Grad-Shafranov equation and separable form}

A hot, axisymmetric magnetized plasma rotating toroidally in a gravitational field must satisfy the generalized Grad-Shafranov equation (Lovelace et al. 1986). To obtain this equation we assume that the axisymmetric magnetic field $\mathbf{B}$ is in the poloidal plane by writing

$$
\mathbf{B}=\nabla \psi \times \nabla \zeta,
$$

where $\zeta$ is the toroidal angle and $\psi$ is the poloidal flux function. We take the velocity $\mathrm{V}$ as toroidal

$$
\mathbf{V}=\Omega R^{2} \nabla \zeta
$$

with $\Omega=c d \Phi / d \psi$ the toroidal rotation frequency, and $R$ being the cylindrical radius from the axis of symmetry. We assume that the magnetic field is frozen into the flow so that $c \nabla \Phi=\mathbf{V} \times \mathbf{B}$ with $\mathbf{B} \cdot \nabla \Phi=0$, then the electrostatic potential $\Phi$ must be a flux function to lowest order. Next, the gravitational potential $G$ is written as

$$
G=-G_{O} M_{O} / r
$$

with $G_{O}$ being the gravitational constant, $r$ is the spherical radius, and $M_{O}$ is the mass of the astrophysical body that is assumed to be a compact source centered at $r=0$, such that $R=r \sin \vartheta$ with $\vartheta$ being the angle from the axis of symmetry. We define our spherical and cylindrical coordinates to satisfy $r \nabla \vartheta=R \nabla \zeta \times \nabla r$ and $\nabla z=R \nabla R \times \nabla \zeta$

The hot magnetized plasma must adjust its flux surfaces to satisfy Ampere's Law, $c \nabla \times \mathbf{B}=4 \pi \mathbf{J}$, and total momentum balance under the influence of the attractive force of gravity and the outward centrifugal and plasma pressure forces,

$$
c^{-1} \mathbf{J} \times \mathbf{B}=\nabla p+M n(\nabla G+\mathbf{V} \cdot \nabla \mathbf{V})=M n\left(\nabla G-\Omega^{2} R \nabla R\right)+\nabla(2 n T),
$$

where $M$ is the mass of the plasma ions, $n$ is their density, and the total pressure of the quasineutral plasma $\left(Z n=n_{\mathrm{e}}=\right.$ electron density) is $p=n\left(T_{\mathrm{i}}+Z T_{\mathrm{e}}\right)=2 n T$, with $T_{\mathrm{i}}$ and $T_{\mathrm{e}}$ being the ion and electron temperatures and $Z$ the charge number of the ions. The relation between $p$ and $n$ defines the effective plasma temperature $T$. This temperature must be a flux function based on the kinetic constraint $\mathbf{B} \cdot \nabla T=0$ that has to be satisfied for a rotating Maxwellian to satisfy the gyro-averaged or drift kinetic equation in the frozen-in limit $c \nabla \Phi=\mathbf{V} \times \mathbf{B}$ (Hinton and Wong 1985; Catto et al. 1987; Helander 2014). 
Importantly, the rotation must satisfy total parallel momentum balance,

$$
\text { B } \cdot \nabla\left[2 G-\Omega^{2} R^{2}+(4 T / M) \ell n(n / \eta)\right]=0,
$$

with $\mathbf{B} \cdot \nabla \eta=0$, so the normalizing density $\eta$ is a flux function. Here $\ell n$ denotes the natural logarithm of $n / \eta$. The density and normalizing or 'pseudo' density are related via the generalized Maxwell-Boltzmann exponential factor that retains the centrifugal and gravitational potentials,

$$
n=n(\psi, \vartheta)=\eta(\psi) e^{\kappa(\psi, \vartheta)}=\eta(\psi) e^{\kappa(\psi, \vartheta)} .
$$

The preceding form for the density conveniently separates poloidal angle and flux function dependences. The parallel momentum constraint determines $\kappa$ within a flux function that can be set to zero by absorbing it into the pseudo-density $\eta$. As a result,

$$
2 u^{2} \kappa=\Omega^{2} R^{2}-2 G,
$$

with $u^{2}=2 T / M$. We see that the flux surfaces and poloidal variation of the density must be allowed to adjust to maintain parallel force balance between the inward central gravity force and the outward cylindrical rotation force.

The $\nabla \zeta$ component of total momentum balance requires $\mathbf{J} \cdot \nabla \psi=0$ and radial Ampere's law requires $\mathbf{J} \cdot \nabla r=0$, giving $\mathbf{J} \cdot \mathbf{B}=0$. As a result, there is only toroidal current,

$$
\mathbf{J}=\left(c M n / B^{2}\right) \nabla \psi \cdot\left[(2 / M n) \nabla(n T)+\nabla G-\Omega^{2} R \nabla R\right] \nabla \zeta .
$$

The toroidal component of Ampere's law then gives the desired Grad-Shafranov form,

$$
\nabla \cdot\left(R^{-2} \nabla \psi\right)=-\left(4 \pi M n / R^{2} B^{2}\right) \nabla \psi \cdot\left[\left(u^{2} / \eta\right) \nabla \eta+\nabla u^{2}+u^{2} \nabla \kappa+\nabla G-\Omega^{2} R \nabla R\right] .
$$

The preceding derivation of the Grad-Shafranov equation generalizes the previous form of Krasheninnikov and Catto (1999) to include rotation. The assumptions used to derive this form satisfy the general constraints for a drifting Maxwellian solution to the drift kinetic or the gyrophase-averaged Fokker-Planck equation in an axisymmetric system (Hinton and Wong 1985; Catto et al. 1987; Helander 2014). In the presence of gravity, the only change is that gravity enters the parallel pressure balance equation (5). These constraints require $\nabla \cdot \mathbf{V}=0$ and $\mathbf{B} \cdot \nabla \mathbf{V} \cdot \mathbf{B}=0$, as well as $\mathbf{B} \cdot \nabla T=0=\mathbf{V} \cdot \nabla T$, and are more restrictive than ideal MHD constraints as they do not allow poloidal flow. The constraints do not require the density, pressure, or entropy density to be flux functions as is sometimes assumed (Lovelace et al. 1986; McClements and Thyagaraya 2001; Throumoulopoulos and Tasso 2001) based on ideal MHD, which allows sonic poloidal flows unlike drift kinetic treatments.

We seek a separable solution of the Grad-Shafranov equation by using the technique introduced in Krasheninnikov et al. (1999) for a point dipole magnetic field confining hot plasma in the absence of gravity and rotation. Subsequent work considered hot, rotating plasma in the absence of gravity (Catto and Krasheninnikov 1999) and hot, stationary plasma confined by gravity (Krasheninnikov and Catto 1999). Here we consider the more general case of a massive localized gravitational source immersed in a magnetic field confining hot, rotating plasma. Unlike Krasheninnikov and Catto (1999) and Krasheninnikov et al. (2000), we find it is unnecessary to introduce a poloidally varying temperature profile. We restrict our attention to a poloidal flux function of the form

$$
\psi=\psi_{O} H(\mu)\left(r_{O} / r\right)^{\alpha},
$$


where $\mu=\cos \vartheta$ and our normalization is $H(\mu=0)=1$. In the vacuum limit, $H=1-\mu^{2}$, and we recover a homogeneous magnetic field for $\alpha=-2$, and the point dipole solution if $\alpha=1$, with $\psi_{O}$ a constant reference value of the flux function at the reference location $r_{O}$ (reference values are denoted by a subscript ' $O$ ' and defined in the equatorial plane $\mu=0$ ). The reference location is arbitrary because of the self-similarity or scale invariance of the solution form of the poloidal flux function.

The magnetic field associated with $\psi$ is then given by

$$
\mathbf{B}=B_{O}\left(\frac{r_{O}}{r}\right)^{\alpha+2}\left[\frac{\nabla r}{\alpha} \frac{d H}{d \mu}-\frac{H r \nabla \vartheta}{\sin \vartheta}\right],
$$

with $B_{O}=-\alpha \psi_{O} / r_{O}^{2}$. For boundary conditions, we demand up-down symmetry,

$$
d H /\left.d \mu\right|_{\mu=0}=0,
$$

and require that the poloidal magnetic field vanishes at the poles,

$$
H(\mu \rightarrow \pm 1) / \sqrt{1-\mu^{2}} \rightarrow 0 .
$$

To obtain an ordinary differential equation for $H$, the expressions $R / r_{O}=$ $\left(\psi_{O} H / \psi\right)^{1 / \alpha}\left(1-\mu^{2}\right)^{1 / 2}$ and $r / r_{O}=\left(\psi_{O} H / \psi\right)^{1 / \alpha}$ suggest taking the Keplerian form,

$$
\Omega^{2}=\Omega_{O}^{2}\left(\psi / \psi_{O}\right)^{3 / \alpha} \propto 1 / r^{3} .
$$

In addition, to make $\kappa$ independent of $\psi$, we take

$$
2 T / M=u^{2}=u_{O}^{2}\left(\psi / \psi_{O}\right)^{1 / \alpha} .
$$

For these choices,

$$
\kappa=\left(\Omega_{O}^{2} r_{O}^{2} / 2 u_{O}^{2}\right)\left(1-\mu^{2}\right) H^{2 / \alpha}+\left(G_{O} M_{O} / r_{O} u_{O}^{2}\right) H^{-1 / \alpha},
$$

where we also define

$$
\kappa_{O}=\left(\Omega_{O}^{2} r_{O}^{2} / 2 u_{O}^{2}\right)+\left(G_{O} M_{O} / r_{O} u_{O}^{2}\right)
$$

and

$$
n_{O}=n\left(\psi=\psi_{O}, \mu=0\right)=\eta_{O} e^{\kappa_{O}} .
$$

Finally, returning to the Grad-Shafranov equation, we obtain an ordinary differential equation for $H$ by taking the density to be

$$
n=\eta e^{\kappa}=n_{O}\left(\eta / \eta_{O}\right) e^{\kappa-\kappa_{O}}=n_{O}\left(\psi / \psi_{O}\right)^{2+3 / \alpha} e^{\kappa-\kappa_{O}} .
$$

Inserting this dependence for $n$ along with our other choices, we are led to define the positive constants,

$$
\begin{gathered}
g=\frac{8 \pi G_{O} M_{O} M n_{O}}{B_{O}^{2} r_{O}}=\left.\frac{8 \pi G_{O} M_{O} M n}{B^{2} r}\right|_{\mu=0}, \\
\omega^{2}=\frac{4 \pi M n_{O} \Omega_{O}^{2} r_{O}^{2}}{B_{O}^{2}}=\left.\frac{4 \pi M n \Omega^{2} r^{2}}{B^{2}}\right|_{\mu=0},
\end{gathered}
$$

and

$$
\beta=\frac{8 \pi M n_{O} u_{O}^{2}}{B_{O}^{2}}=\left.\frac{8 \pi n\left(T_{i}+Z T_{e}\right)}{B^{2}}\right|_{\mu=0}=\left.\frac{8 \pi M n u^{2}}{B^{2}}\right|_{\mu=0},
$$

corresponding to the ratios of the gravitational, rotational, and thermal energies, respectively, normalized by the magnetic energy. As indicated, these parameters are 
constants that do not vary in the equatorial plane. From parallel pressure balance we can see that when the centrifugal force in the equatorial plane is too strong $\left(\Omega^{2} R^{2}>2 G\right.$ or $\left.2 \omega^{2}>g\right)$, the central gravitational force is unable to confine the rotating plasma. However, when the centrifugal force is weaker than the gravitational force $\left(2 G>\Omega^{2} R^{2}\right.$ or $\left.g>2 \omega^{2}\right)$, the pressure of the rotating plasma can adjust flux surfaces via the Grad-Shafranov equation to maintain parallel pressure balance. We find physically interesting equilibrium solutions only in the sub-Keplerian limit, $2 G>\Omega^{2} R^{2}$, but we refer to the motion as Keplerian since $\Omega \propto 1 / r^{3 / 2}$. In addition, equilibrium is impossible without magnetic field or in a cold $(T=0)$ plasma.

Using the preceding definitions, the Grad-Shafranov equation becomes a nonlinear second-order ordinary differential equation for $H$ :

$$
\frac{d^{2} H}{d \mu^{2}}+\frac{\alpha(\alpha+1)}{1-\mu^{2}} H=\alpha\left[\frac{g}{2} H^{-1 / \alpha}-\omega^{2}\left(1-\mu^{2}\right) H^{2 / \alpha}-(\alpha+2) \beta\right] H^{1+4 / \alpha} e^{\kappa-\kappa_{O}} .
$$

A slightly different form for this equation integrated from $\mu=0$ to $\mu=1$ gives a useful integral constraint on $\alpha$,

$$
\begin{gathered}
(\alpha-1)(\alpha+2) \int_{0}^{1} d \mu H=\alpha \int_{0}^{1} d \mu\left(1-\mu^{2}\right) H^{1+4 / \alpha}\left[\frac{g}{2} H^{-1 / \alpha}-\omega^{2}\left(1-\mu^{2}\right) H^{2 / \alpha}\right. \\
-(\alpha+2) \beta] e^{\kappa-\kappa_{O}} .
\end{gathered}
$$

The preceding equations agree with the various cases considered in Catto and Krasheninnikov (1999); Krasheninnikov and Catto (1999); and Krasheninnikov et al. (1999, 2000).

We note that there is a possible problem with singular integrals as the poles are approached due to the $g$ term in $\kappa-\kappa_{\mathrm{O}}$ if $\alpha>0$ (closed flux surface, dipolar solutions) since $H \rightarrow 0$. Therefore, by retaining gravity we are forced to consider $\alpha<0$. We first examine the vacuum root $\alpha=-2$ before focusing more generally on $\alpha<0$. This root corresponds to the limit of a compact object, gravitationally and magnetically confining toroidally rotating hot plasma immersed in a homogeneous magnetic field $\mathbf{B}=B_{O}[\cos \vartheta \nabla r-r \sin \vartheta \nabla \vartheta]=B_{O} \nabla z$. The $\alpha=-2$ root is not altered by finite $\beta$ effects in the absence of rotation and gravity.

For $\alpha<0$, flux surfaces are open to infinity, and, in the presence of gravity when $\alpha>-2$, flare out from the localized source at the origin with the flare vanishing as $\alpha \rightarrow-2$. The behavior of the magnetic field is illustrated schematically in Figs. 1(a)(c). Figure 1(a) shows its behavior for $\alpha \rightarrow-2$, while Fig. 1(b) shows the behavior when $\alpha>-2$ and not approaching zero, and finally Fig. 1(c) gives the field line behavior as $\alpha \rightarrow 0$. All three limits allow an equatorial plane disk (shown shaded).

\section{Equilibrium solutions of the Grad-Shafranov equation}

It is helpful to first observe that for $g=0$ there is an exact solution $H=$ $\left(1-\mu^{2}\right)^{-\alpha / 2}$ to the Grad-Shafranov equation for arbitrary $\beta$, giving an axial field $\mathbf{B}=B_{O}\left(r_{O} / R\right)^{2+\alpha} \nabla z$ and

$$
\alpha+2=-\omega^{2} /(\beta+1)
$$

for $\omega^{2} \sim 1 \sim b$ and $\alpha<0$. However, $\alpha<-2$, so the magnetic field will increase with radius. This axially or cylindrically symmetric $z$-independent solution is not of interest in the presence of gravity, but it suggests that any departure from $z$ 

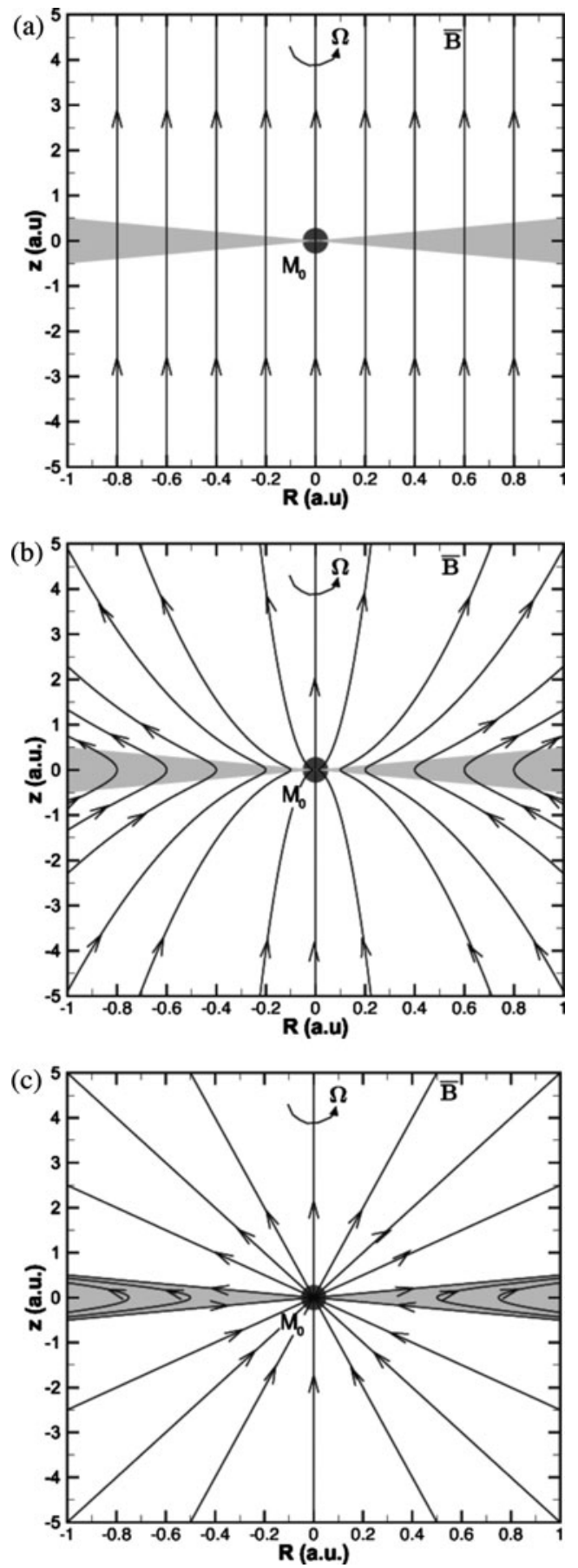

Figure 1. Schematic figures of the magnetic field configurations with equatorial plane disks shaded. (a) A simple axial magnetic field for a plasma at low $\beta$ (the $\beta \ll 1$ or $\alpha \rightarrow-2$ limit). (b) The behavior of the magnetic field as the strong gravitational attraction pinches the magnetic field lines inward in the presence of strong rotation for a plasma at high $\beta(\beta \gg 1)$. (c) The strongly flared magnetic field limit when $\beta \rightarrow \infty$ (the $\alpha \rightarrow 0$ limit). The plasma disk width $\Delta$ scales differently in the various cases: (a) $\Delta=R(2 \beta / g)^{1 / 2}$ for $\beta \ll \mathrm{g} \sim \omega^{2} \ll 1$ and $\Delta=R \beta^{1 / 2} /\left(g-2 \omega^{2}\right)$ for $\mathrm{g}-2 \omega^{2} \gg \beta$; (b) and (c) $\Delta=R \beta^{1 / 2} /\left[\left(g-2 \omega^{2}\right)\left(g-2 \omega^{2}-4 \beta\right)\right]^{1 / 2}$ for $g-2 \omega^{2}>4 \beta$. 
independent cylindrical symmetry must be due to gravity and have $\alpha>-2$, the situation we now focus on.

To avoid solutions with magnetic fields increasing with radius, we first consider the small $g$ and $\omega^{2}$ solution for the $\alpha=-2$ root with $\mathrm{H}=1-\mu^{2}$. Inserting this solution into the integral constraint, we obtain the $g \sim \omega^{2} \ll 1 \sim \beta$ result,

$$
2(\alpha-1)(\alpha+2) / 3=\alpha \int_{0}^{1} d \mu\left[\frac{g}{2} \sqrt{1-\mu^{2}}-\omega^{2}-(\alpha+2) \beta\right] e^{-g\left[1-\sqrt{1-\mu^{2}}\right] / \beta} .
$$

For $\mathrm{g} \ll \beta$ only a weak density departure from cylindrical symmetry is allowed, giving

$$
\alpha \simeq-2+\left[(\pi g / 8)-\omega^{2}\right] /(\beta+1),
$$

where $(\pi g / 8)>\omega^{2}$ is required for a physically interesting solution that keeps $0>\alpha>-2$ and makes the magnetic field fall off with distance and flare slightly. A more interesting limit is $\beta \ll \mathrm{g} \sim \omega^{2} \ll 1$, which requires strong poloidal density variation and for which the integral constraint reduces to

$$
\alpha \simeq-2+\left(g-2 \omega^{2}\right)(\pi \beta / 8 g)^{1 / 2} .
$$

This limit is an extension of the $\omega^{2}=0$ result in Krasheninnikov and Catto (1999) and Krasheninnikov et al. (2000) and requires $g>2 \omega^{2}$ for a physically interesting, slightly flared magnetic field decreasing with radius.

The result for $\beta \ll \mathrm{g} \sim \omega^{2} \ll 1$ has the important feature that the plasma density, and therefore its pressure and the current density, is strongly localized to the equatorial plane since $n / n_{O}=\left(\psi / \psi_{O}\right)^{2+3 / \alpha} e^{-(g / \beta)\left[1-\sqrt{1-\mu^{2}}\right]}$, with a disk thickness $\Delta=R(2 \beta / g)^{1 / 2}$. This plasma disk behavior suggests looking for solutions in the vicinity of the equatorial plane more generally (Lovelace et al. 1986).

To find more general disk solutions allowing the magnetic field to flare while being localized to the equatorial plane, we must consider $g-2 \omega^{2} \gg \beta$ so that the exponential dependence $e^{\kappa-\kappa_{0}}$ in the Grad-Shafranov equation provides the desired localization about $\mu=0$. Then we can find plasma disk solutions with strong poloidal variation. To do so, we begin by approximating the Grad-Shafranov equation by

$$
d^{2} H / d \mu^{2} \simeq \alpha\left[(g / 2)-\omega^{2}-(\alpha+2) \beta\right] e^{(g / \beta)\left[H^{-1 / \alpha}-1\right]+\left(\omega^{2} / \beta\right)\left[H^{2 / \alpha}-1\right]},
$$

where now both rotation and gravity enter the exponential dependence from the density.

To extend our previous results, we note that on the right side of the differential equation for $H$ we are only interested in the region about $H=1$, so we may use

$$
H^{1 / \alpha}=e^{(1 / \alpha) \ell n H} \simeq e^{(1 / \alpha)(H-1)} \simeq 1+(H-1) / \alpha+\ldots
$$

in $e^{\kappa-\kappa_{0}}$ and then assume $g-2 \omega^{2} \gg \beta$ to obtain strong exponential decay away from the equatorial plane. We then obtain

$$
d^{2} H / d \mu^{2} \simeq \alpha\left[(g / 2)-\omega^{2}-(\alpha+2) \beta\right] e^{\left(g-2 \omega^{2}\right)(1-H) / \alpha \beta} .
$$

For $0>\alpha>-2$ and $H \leqslant 1$, we must assume $g>2 \omega^{2}$ to obtain pinched in or flared magnetic field solutions localized about the equatorial plane. Recall that solutions strongly localized to the equatorial plane in the presence of gravity are not possible for $g<2 \omega^{2}$ and $0>\alpha>-2$ since the rotation is too strong for the plasma to be confined gravitationally. 
Multiplying our simplified Grad-Shafranov equation by $d H / d \mu$ and integrating from $H=1$ (at $\mu=0$ ) to $H<1$ (for $\mu^{2}>0$ ) gives

$$
d H / d \mu \simeq \alpha \sqrt{\beta\left(g-2 \omega^{2}\right)\left[1-e^{\left(g-2 \omega^{2}\right)(1-H) / \alpha \beta}\right] /\left[g-2 \omega^{2}-2(\alpha+2) \beta\right]},
$$

where we select the negative root to make $d H / d \mu<0$. To find $H$ we use $\int d x / \sqrt{1-e^{-x}}=2 \tanh ^{-1} \sqrt{1-e^{-x}}$ to obtain

$$
\frac{g-2 \omega^{2}}{\alpha \beta}(H-1)=x=-\ln \left[1-\tanh ^{2}(\sigma \mu / 2)\right] \rightarrow\left\{\begin{array}{cc}
(\sigma \mu / 2)^{2}+\ldots & \sigma|\mu| / 2 \ll 1 \\
\pm \sigma \mu-\ln 4+\ldots & \sigma|\mu| / 2 \gtrsim 1
\end{array},\right.
$$

where $\sigma \equiv \sqrt{\left(g-2 \omega^{2}\right)\left[g-2 \omega^{2}-2(\alpha+2) \beta\right] / \beta}$ and the upper (lower) sign is for $\mu>0(\mu<0)$. Again, $\ell n$ is used to denote the natural logarithm of a quantity. Consequently, for $x=\left(g-2 \omega^{2}\right)(H-1) / \alpha \beta \gg 1$ we obtain a solution strongly localized about the equatorial plane that results in only a small departure from the vacuum solution $H=1-\mu^{2}$ that remains an adequate approximation elsewhere. Moreover, as $\beta$ increases, the $\beta$ dependence of $x=\left(g-2 \omega^{2}\right)(H-1) / \alpha \beta \gg 1$ causes the $H-1$ solution to extend to $\mu^{2}=1$ by satisfying $d^{2} H / d \mu^{2}=0$ since $x=\left(g-2 \omega^{2}\right)(H-1) / \alpha \beta \simeq \pm \sigma \mu$.

Using the preceding approximations for $\mathrm{x} \gg 1$ on the right side of the integral constraint with $z \ll 1$ and the vacuum solution $H=1-\mu^{2}$ inserted on the left side yields,

$$
\begin{aligned}
\frac{4(\alpha-1)(\alpha+2)}{3 \alpha} & \simeq \sqrt{\frac{\beta\left[g-2 \omega^{2}-2(\alpha+2) \beta\right]}{\left(g-2 \omega^{2}\right)}} \int_{z}^{1} d z / \sqrt{1-z} \\
& \simeq 2 \sqrt{\frac{\beta\left[g-2 \omega^{2}-2(\alpha+2) \beta\right]}{\left(g-2 \omega^{2}\right)}}
\end{aligned}
$$

where $z=e^{\left(g-2 \omega^{2}\right)(1-H) / \alpha \beta}=e^{-x}$ and we have assumed $g-2 \omega^{2} \gg-\alpha \beta$ to make the lower limit zero. For $\beta \ll 1$, we recover

$$
\alpha+2=\sqrt{\beta}
$$

as found in Krasheninnikov and Catto (1999) without rotation. In this case we see more generally that gravity and rotation are altering the flux surface shape without explicitly entering the eigenvalue $\alpha$. We are only able to find a solution if $2 \omega^{2}<g$ so that the gravitational force is strong enough to balance the centrifugal force. The localized equatorial plasma disk solution for $\beta \ll 1$ is then $H-1=\mp 2 \mu \sqrt{\beta}$, giving $x= \pm\left(g-2 \omega^{2}\right) \mu / \sqrt{\beta}$ and a plasma disk thickness $\Delta=R \beta^{1 / 2} /\left(g-2 \omega^{2}\right)$, and requiring $g-2 \omega^{2} \gg \sqrt{\beta}$.

As $\beta$ becomes larger, the $\beta$ dependence of $x=\left(g-2 \omega^{2}\right)(H-1) / \alpha \beta \simeq \pm \sigma \mu$ allows the $x \gg 1$ solution to extend over the entire plasma as illustrated schematically in Fig. 1(b). Again, assuming $g-2 \omega^{2} \gtrsim-\alpha \beta$, we consider $0<-\alpha \ll 2$, and note that for

$$
H-1= \pm \alpha \mu \sqrt{\beta\left(g-2 \omega^{2}-4 \beta\right) /\left(g-2 \omega^{2}\right)}
$$

to vanish at $\mu= \pm 1$, we need $H-1=\mp \mu$, giving

$$
0<-\alpha \simeq \sqrt{\left(g-2 \omega^{2}\right) /\left[\beta\left(g-2 \omega^{2}-4 \beta\right)\right]} \ll 2,
$$

a result that can also be found from the integral constraint, and is consistent with the $\omega^{2}=0$ and $g \gg \beta$ result of Krasheninnikov and Catto (1999). We require $g-2 \omega^{2}>$ 
$4 \beta \gg 1$, giving $x= \pm \mu \sqrt{\left(g-2 \omega^{2}\right)\left(g-2 \omega^{2}-4 \beta\right) / \beta}$ and $\Delta=R \beta^{1 / 2} /[(g-$ $\left.\left.2 \omega^{2}\right)\left(g-2 \omega^{2}-4 \beta\right)\right]^{1 / 2}$ to be the disk width.

These results indicate that once $g-2 \omega^{2}>4 \beta \gg 1$, the currents associated with gravity, rotation, and diamagnetism become localized to the equatorial disk in such a way as to cause equatorial plane pinching and thereby strong flaring in the magnetic field. In particular, when $\beta \rightarrow \infty$ so that $\alpha \rightarrow 0$, the strongly flared magnetic field becomes nearly radial outside the equatorial plane, where it is given by

$$
\mathbf{B}=B_{O}\left(r_{o} / r\right)^{2}\left[\mp \alpha^{-1} \nabla r-r \sqrt{(1 \mp \mu) /(1 \pm \mu)} \nabla \vartheta\right] .
$$

This expression fails right at the equatorial plane since the $\sigma|\mu| \gtrsim 1$ expansion breaks down at very small $\mu$. Within the plasma disk, the magnetic field has an axial component. This limit is shown schematically in Fig. 1(c).

Our $\beta \gg 1$ limit can also be examined for larger $-\alpha$ by picking $\alpha$ such that $H-1=. \mp \mu= \pm \alpha \mu \sqrt{\beta\left[g-2 \omega^{2}-2(\alpha+2) \beta\right] /\left(g-2 \omega^{2}\right)}$. Then $\alpha=-1$ for $g-$ $2 \omega^{2}=2 \beta^{2} /(\beta-1)$ and we have a thick disk of width $\Delta=R \beta^{1 / 2} /\left[\left(g-2 \omega^{2}\right)(g-\right.$ $\left.\left.2 \omega^{2}-2 \beta\right)\right]^{1 / 2}=R(\beta-1) / 2 \beta$. In this case the magnetic field falls off more slowly, as $1 / r$, and has significant flaring outside the equatorial plane, somewhat similar to that found by Ogilvie (1997):

$$
\mathbf{B}=B_{O}\left(r_{o} / r\right)[ \pm \nabla r-r \sqrt{(1 \mp \mu) /(1 \pm \mu)} \nabla \vartheta] .
$$

Similarly, $\alpha=-1 / 2$ for $g-2 \omega^{2}=3 \beta^{2} /(\beta-4)$, while $\alpha=-3 / 2$ for $g-2 \omega^{2}=$ $\beta^{2} /(\beta-4 / 9)$.

\section{Discussion}

We find a realistic rotating and magnetized three-dimensional axisymmetric equilibria for hot plasma confined by a gravitational field. The plasma density and current exhibit strong localization to the equatorial plane, resulting in plasma disk equilibria with open magnetic field lines. Dipole equilibria are not possible within the model. Our most important results are for $g-2 \omega^{2} \gtrsim \beta \gg 1$, and for $g-2 \omega^{2} \gg \sqrt{\beta}$ and $\beta \ll 1$. The small $\beta$ limit only allows small departures from a cylindrically symmetric homogeneous magnetic field in which the plasma is strongly localized to the equatorial plane. In this limit only weakly flared, open flux surfaces are obtained with the magnetic field 'pinched in' at the equatorial plane. The pinching in at the equatorial plane that results in the flare of the open field lines is an indication that the frozen-in plasma on the ambient magnetic field lines is being gravitationally squeezed toward the massive localized gravitational source. Our large $\beta$ results lead to substantially more flaring of magnetic field due to the strong localization of plasma to the equatorial plane, with the magnetic field even becoming strongly radial outside the equatorial disk as $\beta \rightarrow \infty$. This magnetic field is strongest at the poles, but the magnetic energy is much less than the gravitational, rotational, and plasma energies. However, even though the magnetic field is weak, it is needed to allow the strong poloidal density variation that acts to maintain parallel pressure balance as the radial gravitational and cylindrical centrifugal forces become misaligned outside the equatorial plane. Unlike Blandford and Payne (1982), our results do not indicate any restriction on the angle that the poloidal field makes with the equatorial plane. However, their treatment allows poloidal flow along the magnetic field lines that is not allowed by our kinetic constraints (Hinton and Wong 1985; Catto et al. 1987; Helander 2014). 
Our open magnetic field model is admittedly simple, and its simplicity is appealing and very likely a virtue, since it makes predictions on the plasma rotation frequency bound $\left(g>2 \omega^{2}\right.$ for $\beta \ll 1$ and $g>2 \omega^{2}+\beta$ for $\left.\beta \gg 1\right)$, the condition for having a plasma disk in the equatorial plane $(g \gg \sqrt{\beta}$ for $\beta \ll 1$ and $g \gg \beta$ for $\beta \gg 1$ ), and the requirement for strong magnetic field flaring $(g \gg \beta \gg 1)$ that may be possible to check. In our model, no equilibria can be found if gravity is too weak $\left(g<2 \omega^{2}\right.$ for $\beta \ll 1$ or $g<2 \omega^{2}+\beta$ for $\beta \gg 1$ ), since then the gravitational force is unable to balance the centrifugal force and the plasma pressure.

The Keplerian rotation frequency of our model can only be directly related to the rotation frequency of the localized gravitational source if co-rotation is possible. Then the logical reference radius is the surface of the massive source. However, at small radii, reconnection may take place to prevent the co-rotation of magnetic field and a toroidal magnetic field may be generated. It is also important to realize that accretion is not considered because it happens on the slower time scales associated with radiative cooling and momentum transport.

Finally, our model has motivated us to reconsider magneto-rotational stability (Velikhov 1959; Balbus and Hawley 1991) for a self-consistent equilibrium. These investigations are underway but incomplete at this time. However, in the axial magnetic field limit the stability threshold is found to be sensitive to compressibility effects, density variation, and gravity as well as the Keplerian radial variation of the toroidal rotation frequency of the plasma. Moreover, unlike the standard result, the stability threshold in the presence of gravity is found to be rather different than when it is absent unless the standard strict Keplerian assumption $2 G=\Omega^{2} R^{2}$ is employed.

\section{Acknowledgements}

Peter J. Catto thanks Merton College and Peierls Centre for Theoretical Physics at Oxford for rekindling interest in this problem and for time for preliminary considerations. He is particularly grateful and indebted to Alex Schekochihin of Merton College at Oxford who arranged the visit and provided incomparable hospitality. Peter J. Catto also thanks Jeff Freidberg for motivating him to return to the problem after returning home. Finally, the authors thank Silvia Espinosa for her artistic contributions. Work supported by the US Department of Energy grants DE-FG02-91ER-54109 at MIT and DE-FG02-04ER54739 at UCSD.

\section{REFERENCES}

Balbus, S. A. and Hawley, J. F. 1991 A powerful local shear instability in weakly magnetized disks. I. Linear analysis. Astrophys. J. 376, 214-222.

Blandford, R. D. and Payne, D. G. 1982 Hydromagnetic flows from accretion discs and the production of radio jets. Mon. Not. R. Astr. Soc. 199, 883-903.

Catto, P. J., Bernstein, I. B. and Tessarotto, M. 1987 Ion transport in toroidally rotating tokamak plasmas. Phys. Fluids 30, 2784-2795.

Catto, P. J. and Krasheninnikov, S. I. 1999 Effects of rotation on a finite plasma pressure equilibrium in a dipolar magnetic field. Phys. Lett. A 258, 153-157.

Chandrasekhar, S. 1956 Axisymmetric magnetic fields and fluid motions. Ap. J. 124, 232-243.

Ghanbari, J. and Abbassi, S. 2004 Equilibria of a self-gravitating, rotating disc around a magnetized compact object. Mon. Not. Roy. Astr. Soc. 350, 1437-1444.

Helander, P. 2014 Theory of plasma confinement in non-axisymmetric magnetic fields. Rep. Prog. Phys. 77, 087001-087035.

Hinton, F. L. and Wong, S. K. 1985 Neoclassical ion transport in rotating axisymmetric plasmas. Phys. Fluids 28, 3082-3098. 
Krasheninnikov, S. I. and Catto, P. J. 1999 Equilibrium of a gravitating plasma in a dipolar magnetic field. Phys. Lett. A 260, 502-506.

Krasheninnikov, S. I., Catto, P. J. and Hazeltine, R. D. 1999 A magnetic dipole equilibrium solution at finite plasma pressure. Phys. Rev. Lett. 82, 2689-2692.

Krasheninnikov, S. I., Catto, P. J. and Hazeltine, R. D. 2000 Plasma equilibria in dipolar magnetic configurations. Phys. Plasmas 7, 1831-1838.

Lovelace, R. V. E., Mehanian, C., Mobarry, C. M. and Sulkanen, M. E. 1986 Theory of axisymmetric magnetohydrodynamic flows: disks. Ap J. S. 62, 1-37.

McClements, K. C. and Thyagaraya, A. 2001 Azimuthally symmetric magnetohydrodynamic and two-fluid equilibria with arbitrary flows. Mon. Not. Roy. Astr. Soc. 323, 733-742.

Ogilvie, G. I. 1997 The equilibrium of a differentially rotating disc containing a poloidal magnetic field. Mon. Not. R. Astron. Soc. 288, 63-77.

Prasanna, A. R., Tripathy, S. C. and Das, A. C. 1989 Equilibrium structure for a plasma magnetosphere around compact objects. J. Astrophys. Astr. 10, 21-34.

Throumoulopoulos, G. N. and Tasso, H. 2001 Axisymmetric equilibria of a gravitating plasma with incompressible flows. Geophys. Astroph. Fluid Dyn. 94, 249-262.

Velikhov, E. P. 1959 Stability of an ideally conducting liquid flowing between cylinders rotating in a magnetic field. Sov. Phys. JETP 36, 995-998. 